\title{
The Impact of Different Types of Shift Work on Blood Pressure and Hypertension: A Systematic Review and Meta-Analysis
}

\author{
Sara Gamboa Madeira ${ }^{1,2, * \mathbb{D}}$, Carina Fernandes ${ }^{3,4}$, Teresa Paiva ${ }^{5,6}$, Carlos Santos Moreira ${ }^{7}$ \\ and Daniel Caldeira ${ }^{8,9,10}$
}

Citation: Gamboa Madeira, S. Fernandes, C.; Paiva, T.; Santos Moreira, C.; Caldeira, D. The Impact of Different Types of Shift Work on Blood Pressure and Hypertension: A Systematic Review and Meta-Analysis. Int. J. Environ. Res. Public Health 2021, 18, 6738. https:/ / doi.org/10.3390/ijerph18136738

Academic Editors: Tae-Won Jang, Hyoung-Ryoul Kim, Mo-Yeol Kang and Hye-Eun Lee

Received: 24 May 2021

Accepted: 17 June 2021

Published: 23 June 2021

Publisher's Note: MDPI stays neutral with regard to jurisdictional claims in published maps and institutional affiliations.

Copyright: (C) 2021 by the authors. Licensee MDPI, Basel, Switzerland. This article is an open access article distributed under the terms and conditions of the Creative Commons Attribution (CC BY) license (https:// creativecommons.org/licenses/by/ $4.0 /)$.
1 Instituto de Saúde Ambiental (ISAMB), Faculdade de Medicina, Universidade de Lisboa, 1649-026 Lisbon, Portugal

2 Family Health Unit Mactamã, Administração Regional de Saúde de Lisboa e Vale do Tejo, 2745-862 Lisbon, Portugal

3 Escola Nacional de Saúde Pública, Universidade Nova de Lisboa, 1600-560 Lisbon, Portugal; fernandes.carina@gmail.com

4 Neurology Department, Hospital das Forças Armadas, 1649-020 Lisbon, Portugal

5 Sleep Medicine Center (CENC), 1070-068 Lisbon, Portugal; teresapaiva0@gmail.com

6 Comprehensive Health Research Center (CHRC), Nova Medical School, Universidade Nova de Lisboa, 1169-056 Lisbon, Portugal

7 Medicine Clinic I, Faculdade de Medicina, Universidade de Lisboa, 1649-028 Lisbon, Portugal; carlos.moreira@medicina.ulisboa.pt

8 Cardiology Department, Hospital de Santa Maria/Santa Maria University Hospital—Centro Hospitalar Universitário Lisboa Norte (CHULN), 1649-028 Lisbon, Portugal; dgcaldeira@hotmail.com

9 Laboratory of Clinical Pharmacology and Therapeutics, Faculdade de Medicina, Universidade de Lisboa, 1649-028 Lisbon, Portugal

10 Centro Cardiovascular da Universidade de Lisboa (CCUL), CAML, Faculdade de Medicina, Universidade de Lisboa, 1649-028 Lisbon, Portugal

* Correspondence: smadeira@medicina.ulisboa.pt

\begin{abstract}
Shift work (SW) encompasses 20\% of the European workforce. Moreover, high blood pressure (BP) remains a leading cause of death globally. This review aimed to synthesize the magnitude of the potential impact of SW on systolic blood pressure (SBP), diastolic blood pressure (DBP) and hypertension (HTN). MEDLINE, EMBASE and CENTRAL databases were searched for epidemiological studies evaluating BP and/or HTN diagnosis among shift workers, compared with day workers. Random-effects meta-analyses were performed and the results were expressed as pooled mean differences or odds ratios and 95\% confidence intervals (95\% CI). The Newcastle-Ottawa Scale was used to assess the risk of bias. Forty-five studies were included, involving 117,252 workers. We found a significant increase in both SBD and DBP among permanent night workers $(2.52 \mathrm{mmHg}$, 95\% CI $0.75-4.29$ and $1.76 \mathrm{mmHg}, 95 \%$ CI $0.41-3.12$, respectively). For rotational shift workers, both with and without night work, we found a significant increase but only for SBP $(0.65 \mathrm{mmHg}$, 95\% CI 0.07-1.22 and $1.28 \mathrm{mmHg}, 95 \%$ CI 0.18-2.39, respectively). No differences were found for HTN. Our findings suggest that SW is associated with an increase of BP, mainly for permanent night workers and for SBP. This is of special interest given the large number of susceptible workers exposed over time.
\end{abstract}

Keywords: cardiovascular disease; blood pressure; occupational health; work schedule; permanent shift; rotating shift; night shift; systematic review

\section{Introduction}

Hypertension (HTN) is a major preventable cause of cardiovascular diseases (CVDs) and all-cause mortality in the European continent, with an overall prevalence of 30-45\% [1]. There is a relationship between blood pressure (BP) and CVD events [2], and BP decrease in hypertensive patients has shown to improve the prognosis [3]. Guidelines on CVD prevention stress the importance of a holistic approach, including non-traditional risk 
factors such as socioeconomic status and occupational factors [4]. Shift work (SW) plays an important role in the "24/7" modern societies, involving about $20 \%$ of the European and the American workforces [5]. However, this work arrangement frequently disrupts sleepwake cycle and circadian rhythms, which may affect cardiovascular function including BP. Since shift work is a growing societal trend and high BP a leading risk factor for cardiovascular diseases, it is crucial to clarify the potential impact of shift work, especially when robust data is lacking. The single previous systematic review in this topic focused only on the HTN risk and used heterogeneous definitions for HTN diagnosis and simplistic SW categorization [6]. Therefore, we aimed to determine not only the HTN risk but also the magnitude of BP change among shift workers in comparison with day workers.

\section{Materials and Methods}

This systematic review was conducted in accordance with PRISMA (Preferred Reporting Items for Systematic Reviews and Meta-Analysis) guidelines [7] and its protocol was registered (Available online: https:/ / osf.io/m47qc (Accessed on 24 May 2021)).

\subsection{Literature Search and Selection}

A literature search was performed by personnel experienced in designing strategies for systematic reviews in health sciences databases. The search was performed in MEDLINE, EMBASE and The Cochrane Library electronic database (CENTRAL), on 18 February 2019. There were no limits regarding year of publication, language, study design or geographic origin. Animal studies were excluded. The search strategy is detailed on the supplementary material (Table S1). Two reviewers (SGM and CF) independently evaluated the title and abstract of the retrieved papers to determine if these met the inclusion criteria, using a pre-piloted form. Studies fulfilling the inclusion criteria and those uncertain were analyzed in full-text independently by the two reviewers. At this stage we only considered articles published in English and the reasons for exclusion were recorded. Abstracts and conference papers were excluded. Disagreements were solved through consensus or using a third party (DC).

\subsection{Inclusion Criteria}

We included studies that reported data about BP values and/or diagnosis of HTN in both shift workers and a control group of day workers. We were lenient and broad regarding the definition of shift work, therefore we considered any shift provided if represented a nonstandard schedule, excluding long work hours (e.g., weekend work). If studies reported BP values, we sought the systolic and/or diastolic BP mean values and standard deviation (or other measurement of variability), in both groups. Data from linear regression models on $\mathrm{BP}$ values $(\mathrm{mmHg})$, reporting a $\beta$ coefficient and $95 \% \mathrm{CI}$, were also considered. HTN diagnosis was recorded when it was established using the cut-off values of the current European Guidelines (i.e., systolic BP $\geq 140 \mathrm{mmHg}$ and/or diastolic BP $\geq 90 \mathrm{mmHg}$ in office) [1]. HTN diagnosis was also considered when the subject was under anti-hypertensive medication. Studies in which this diagnosis relied on subjects' self-report or those having other HTN definition thresholds were excluded. Data from binary logistic regression models, reporting estimation of risk (e.g., odds ratio) were included. Studies enrolling exclusively special populations (e.g., pregnant women or clinical populations) and laboratory protocols were excluded since our focus was on "reallife" settings. Additionally, when different papers included, either totally or partially, the same subjects, we selected the study which more accurately and comprehensively answered our research question. For more details see the supplementary material (Table S2).

\subsection{Data Extraction}

Data was independently extracted from the included studies by two reviewers (SGM and CF) into a standardized form. Disagreements were solved through consensus. The following data were extracted: study design and follow-up (for longitudinal studies), 
occupational setting, sample size, mean age, sex, shift work schedule definition and source of information and method of BP assessment. For outcomes, systolic and diastolic BP mean and standard deviation or standard error, HTN diagnosis, effect size measurements with 95\% confidence intervals and confounding variables. Adjusted risk estimates were preferred. When more than one regression model was presented, the one that best fitted our research question was included.

\subsection{Methodologic Quality Assessment}

The methodologic quality assessment was also performed independently by two reviewers (SGM and CF). Included studies were graded according to the adequate version of the Newcastle-Ottawa Quality Assessment Scale (NOS) [8,9]. This tool evaluates three dimensions (selection, comparability and outcome), distributed across eight items. A maximum of one point for each item within the "Selection" and "Outcome" categories and maximum of two points for "Comparability" can be given. Higher scores represent a higher methodologic quality; less than 5 points was considered as low quality/high risk of bias [9]. For outcome assessment in cohort studies, the adequate follow-up was defined as 5 years, based on the dose-response relationship between shift work and cardiovascular outcomes suggested in previous studies [10].

\subsection{Data Analysis}

For analysis purpose, we defined categories of SW considering 4 types: permanent night shifts $(\mathrm{PN})$, rotational shifts including nights $(\mathrm{R}+\mathrm{N})$, rotational shifts without nights (RN) and an additional category for the remainder (NS; "Not Specified"). Studies that included several types of SW (e.g., permanent night workers and rotational shifts including nights) were considered independent entries and included in independent meta-analyses.

Pooled mean difference and 95\% CI were estimated for continuous outcomes (systolic $\mathrm{BP}$ and diastolic BP) to quantify the difference in means between each SW type and controls. Pooled odds ratio (OR) and 95\% CI were determined for the dichotomous variable (HTN diagnosis), through random-effects models. The statistical analyses were performed using RevMan 5.4 software (The Nordic Cochrane Centre, The Cochrane Collaboration). Heterogeneity of the pooled effect size estimates was assessed through the $\mathrm{I}^{2}$ statistic to quantify the proportion of the total variation across studies that resulted from heterogeneity rather than chance. Publication bias was assessed through visual inspection of funnel plot asymmetry (see supplementary material-Figure S1) and, also, by Egger test.

Whenever more than ten studies were involved in the meta-analysis of continuous outcomes variables (i.e., SBP and DBP) [11] a meta-regression analysis was performed in order to assess if specific factors (covariates) influence the magnitude of the estimate of effect estimate across studies [11,12]. Similarly to what has been conducted in previous studies on this topic [13], we include covariates related to participants characteristics such as sex (proportion of males) and age (mean values) but also important cardiovascular risk factors such as smoking (proportion of smokers) and body mass index (BMI; average values). We performed univariate and multivariate meta-regression analysis.

\section{Results}

\subsection{Search Results}

Of the 1336 articles retrieved from the electronic database search, 117 underwent fulltext assessment. At full-text appraisal, 72 studies were excluded (Figure 1). At this stage, retrieval of conference abstracts, lacking a full-text article, lead to their exclusion (labelled as "abstract only"). When the same population was used in different studies, only one of the studies was selected (the exclusion was labelled as "duplicate"; more detailed information is provided in the supplementary material-Table S2). Forty-five independent studies met the inclusion criteria. Of these, 41 were included in the meta-analysis for systolic BP, 39 for diastolic BP and 14 for HTN diagnosis (Figure 1). A total of 117,252 workers were implicated, 46,345 of which shift workers (SWs) and 70,907 daytime workers (DWs). 


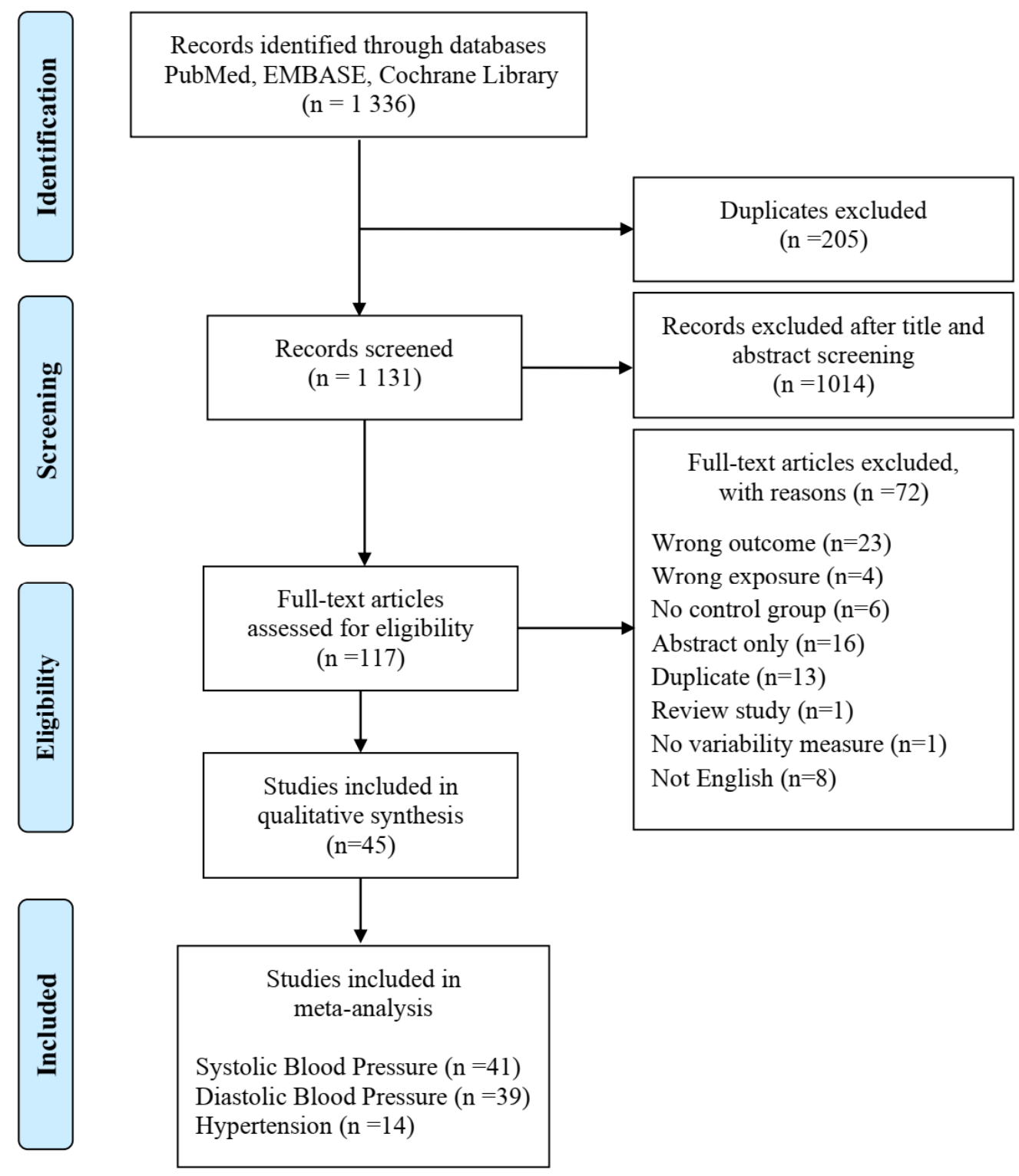

Figure 1. PRISMA flow diagram of literature search, screening and eligibility of the included studies in the meta-analysis.

\subsection{Study Characteristics}

Main characteristics of the 45 included studies [14-58] are presented in Table 1.

Most studies had a cross-sectional design or provided only cross-sectional information. Three studies provided longitudinal data, two being retrospective cohorts $[17,36]$ and one a prospective cohort [49]. The follow-up periods ranged from 10 to 31 years. Most studies were settled in Asia $(n=21)$, mostly in Japan, followed by Europe $(n=13)$, America $(n=9)$ and, lastly, Africa $(n=2)$. Industry was the most frequent occupational setting $(n=25)$, followed by transportation $(n=4)$ and nursing staff $(n=4)$. Nevertheless, the specific job performed by the participants was not always explicit, both for SWs and DWs. In six studies, the authors highlighted that the SWs were mainly blue-collar workers (e.g., machine operators) while DWs were mainly white-collar (e.g., administrative). Sample sizes ranged from 47 to 26,463 participants. Most studies included only male workers $(n=26)$, while 9 studies addressed only females and 10 studies incorporated both sexes. Overall, the participants' mean age was 39.61 years, specifically, 39.64 for SWs and 39.58 for DWs. 
Table 1. Main characteristics of the 45 included studies.

\begin{tabular}{|c|c|c|c|c|c|c|c|c|c|c|}
\hline $\begin{array}{l}\text { Author } \\
\text { Year }\end{array}$ & Design & Country & Population & Sex & Shift Work & $\begin{array}{l}\text { Sample Size } \\
\text { (SWs/DWs) }\end{array}$ & $\begin{array}{l}\text { Mean Age } \\
\text { (SWs/DWs) }\end{array}$ & Outcome & Outcome Adjustments & NOS \\
\hline $\begin{array}{c}\text { Asare-Anane } \\
2015 \\
{[14]}\end{array}$ & CS & Ghana & cocoa industry & $\mathrm{F} \& \mathrm{M}$ & NS & $113 / 87$ & $42.0 / 40.3$ & $\begin{array}{l}\text { SBP } \\
\text { DBP }\end{array}$ & No & 4 \\
\hline $\begin{array}{c}\text { Attarchi } \\
2012 \\
{[15]}\end{array}$ & CS & Iran & $\begin{array}{c}\text { tire } \\
\text { manufacturing } \\
\text { factory }\end{array}$ & M & NS & $88 / 76$ & $38.5 / 40.2$ & $\begin{array}{l}* \mathrm{SBP} \\
{ }^{*} \mathrm{DBP}\end{array}$ & $\begin{array}{c}\text { * age, BMI, smoking, } \\
\text { salt, exercise, family } \\
\text { HTN, job duration }\end{array}$ & 8 \\
\hline $\begin{array}{l}\text { Biggi } \\
2008 \\
{[17]}\end{array}$ & $\begin{array}{c}\mathrm{CH} \\
(76-07)\end{array}$ & Italy & $\begin{array}{l}\text { street cleaning } \\
\text { and waste } \\
\text { collection }\end{array}$ & M & $\mathrm{PN}$ & $331 / 157$ & $47.0 / 42.3$ & $\begin{array}{l}* \mathrm{HTN} \\
* * \mathrm{SBP} \\
* * \mathrm{DBP}\end{array}$ & $\begin{array}{l}\text { * age, job } \\
\text { company and branch, } \\
\text { study period } * * \text { plus } \\
\text { smoking and alcohol }\end{array}$ & 8 \\
\hline $\begin{array}{c}\text { Bursey } \\
1990 \\
{[18]}\end{array}$ & CS & UK & $\begin{array}{l}\text { nuclear fuel } \\
\text { factory }\end{array}$ & $\mathrm{M}$ & $\mathrm{R}+\mathrm{N}$ & $57 / 57$ & $50 / 50$ & $\begin{array}{l}\text { SBP } \\
\text { DBP }\end{array}$ & No & 5 \\
\hline $\begin{array}{l}\text { Chan } \\
1993 \\
{[19]}\end{array}$ & CS & Singapore & $\begin{array}{l}\text { electronics } \\
\text { industry }\end{array}$ & $\mathrm{F}$ & $\begin{array}{c}\mathrm{R}+\mathrm{N} \\
\mathrm{PN}\end{array}$ & $\begin{array}{l}\mathrm{R}+\mathrm{N} 55 / 75 \\
\mathrm{PN} \mathrm{B}^{7} / 63 \\
\mathrm{PN}^{\mathrm{C}} 58 / 59\end{array}$ & $\begin{array}{l}\mathrm{R}+\mathrm{N} 28 / 30 \\
\mathrm{PN}{ }^{\mathrm{B}, \mathrm{C}} \text { NotR }\end{array}$ & $\begin{array}{l}\text { SBP } \\
\text { DBP } \\
\text { HTN }\end{array}$ & No & 4 \\
\hline $\begin{array}{l}\text { Chen } \\
2010 \\
{[20]}\end{array}$ & CS & Taiwan & $\begin{array}{l}\text { semiconductor } \\
\text { manufacturing }\end{array}$ & $\mathrm{F}$ & $\mathrm{PN}$ & $561 / 656$ & $32.7 / 34.9$ & $\begin{array}{l}\text { SBP } \\
\text { DBP }\end{array}$ & No & 4 \\
\hline $\begin{array}{c}\text { De Bacquer } 2009 \\
\text { [21] }\end{array}$ & CS & Belgium & $\begin{array}{l}\text { nine companies } \\
\text { and public } \\
\text { administration }\end{array}$ & M & $\mathrm{R}+\mathrm{N}$ & $309 / 1220$ & $44.7 / 43.1$ & $\begin{array}{l}\text { SBP } \\
\text { DBP }\end{array}$ & No & 6 \\
\hline $\begin{array}{c}\text { Gaudemaris } \\
2011 \\
{[22]}\end{array}$ & CS & France & nursing staff & $\mathrm{F}$ & $\begin{array}{l}\text { PN } \\
\text { NS }\end{array}$ & $\begin{array}{c}\text { PN } 149 \\
\text { NS } 1802 / 1863\end{array}$ & NotR & $\begin{array}{l}\text { SBP } \\
\text { DBP }\end{array}$ & No & 6 \\
\hline $\begin{array}{c}\text { Di Lorenzo } 2003 \\
\text { [23] }\end{array}$ & CS & Italy & chemical industry & M & $\mathrm{R}+\mathrm{N}$ & $185 / 134$ & $48.7 / 48.9$ & $\begin{array}{l}\text { SBP } \\
\text { DBP }\end{array}$ & No & 6 \\
\hline
\end{tabular}


Table 1. Cont.

\begin{tabular}{|c|c|c|c|c|c|c|c|c|c|c|}
\hline $\begin{array}{l}\text { Author } \\
\text { Year }\end{array}$ & Design & Country & Population & Sex & Shift Work & $\begin{array}{l}\text { Sample Size } \\
\text { (SWs/DWs) }\end{array}$ & $\begin{array}{l}\text { Mean Age } \\
\text { (SWs/DWs) }\end{array}$ & Outcome & Outcome Adjustments & NOS \\
\hline $\begin{array}{c}\text { Ely } \\
1986 \\
{[24]}\end{array}$ & CS & US & police officers & M & $\begin{array}{c}\mathrm{R}+\mathrm{N} \\
\mathrm{PN}\end{array}$ & $\begin{array}{c}\mathrm{R}+\mathrm{N} 41 \\
\mathrm{PN} 80 / 156\end{array}$ & $\begin{array}{c}\mathrm{R}+\mathrm{N} 37.4 \\
\mathrm{PN} 38.1 / 40.0\end{array}$ & $\begin{array}{l}\text { SBP } \\
\text { DBP }\end{array}$ & No & 6 \\
\hline $\begin{array}{l}\text { Ohlander } \\
2015 \\
{[25]}\end{array}$ & CS & Germany & car manufacturing & $\mathrm{F} \& \mathrm{M}$ & $\begin{array}{c}\mathrm{R}+\mathrm{N} \\
\mathrm{R}-\mathrm{N} \\
\mathrm{PN}\end{array}$ & $\begin{array}{c}\mathrm{R}+\mathrm{N} 198 \\
\mathrm{R}-\mathrm{N} 9572 \\
\mathrm{PN} \\
3568 / 12,005\end{array}$ & $\begin{array}{c}\mathrm{R}+\mathrm{N} 40.0 \\
\mathrm{R}-\mathrm{N} 38.3 \\
\mathrm{PN} 41.4 / 37.8\end{array}$ & $\begin{array}{c}\text { SBP } \\
\text { DBP } \\
* \text { HTN }\end{array}$ & $\begin{array}{l}\text { *age, sex, BMI, lipids, } \\
\text { smoking, alcohol, } \\
\text { exercise, } \\
\text { sleep disorders, job } \\
\text { status, noise, heat, } \\
\text { social disruption }\end{array}$ & 8 \\
\hline $\begin{array}{c}\text { Fesharaki } \\
2014 \\
{[26]}\end{array}$ & CS & Iran & $\begin{array}{l}\text { steel and } \\
\text { polyacryl } \\
\text { companies }\end{array}$ & $\mathrm{M}$ & $\begin{array}{c}\mathrm{R}+\mathrm{N} \\
\mathrm{R}-\mathrm{N}\end{array}$ & $\begin{array}{c}R+N 4050 \\
R-N 597 / 3966\end{array}$ & $\begin{array}{c}\mathrm{R}+\mathrm{N} 41.62 \\
\mathrm{R}-\mathrm{N} 43.31 / 41.33\end{array}$ & $\begin{array}{l}* \text { SBP } \\
* \text { DBP }\end{array}$ & $\begin{array}{c}\text { *age, BMI, education, } \\
\text { work experience, } \\
\text { marital status }\end{array}$ & 8 \\
\hline $\begin{array}{l}\text { Guo } \\
2013 \\
{[27]}\end{array}$ & CS & China & motor corporation & $\mathrm{F} \& \mathrm{M}$ & $\mathrm{R}+\mathrm{N}$ & $9118 / 17,345$ & $62.4 / 64.22$ & $\begin{array}{l}\text { SBP } \\
\text { DBP }\end{array}$ & No & 6 \\
\hline $\begin{array}{c}\text { Ishizuka } \\
1993 \\
\text { [29] }\end{array}$ & CS & Japan & machine plant & $\mathrm{M}$ & $\mathrm{R}+\mathrm{N}$ & $38 / 21$ & $31.6 / 36.9$ & $\begin{array}{l}\text { SBP } \\
\text { DBP }\end{array}$ & No & 5 \\
\hline $\begin{array}{l}\text { Jermendy } \\
2012 \\
{[30]}\end{array}$ & CS & Hungary & $\begin{array}{c}\text { multiple } \\
\text { occupations }\end{array}$ & $\mathrm{F} \& \mathrm{M}$ & $\mathrm{R}+\mathrm{N}$ & $\begin{array}{c}\text { M 54/67 } \\
\text { F 180/180 }\end{array}$ & $\begin{array}{c}\text { M 42.2/42.5 } \\
\text { F 44.5/42.9 }\end{array}$ & $\begin{array}{l}\text { SBP } \\
\text { DBP }\end{array}$ & No & 4 \\
\hline $\begin{array}{c}\text { Kantermann } \\
2013 \\
{[31]}\end{array}$ & CS & Belgium & steel factory & $\mathrm{M}$ & $\mathrm{R}+\mathrm{N}$ & $32 / 15$ & $39.5 / 45.0$ & $\begin{array}{l}\text { SBP } \\
\text { DBP }\end{array}$ & No & 4 \\
\hline $\begin{array}{c}\text { Kawabe } \\
2014 \\
{[32]}\end{array}$ & CS & Japan & $\begin{array}{l}12 \text { large } \\
\text { companies }\end{array}$ & $\mathrm{F} \& \mathrm{M}$ & $\begin{array}{c}\mathrm{R}+\mathrm{N} \\
\mathrm{R}-\mathrm{N} \\
\mathrm{PN}\end{array}$ & $\begin{array}{c}\mathrm{R}+\mathrm{N} 243 \\
\mathrm{R}-\mathrm{N} 1017 \\
\mathrm{PN} 73 / 3094\end{array}$ & $\begin{array}{c}\mathrm{R}+\mathrm{N} 40.1 \\
\mathrm{R}-\mathrm{N} 37.9 \\
\mathrm{PN} 50.8 / 42.6\end{array}$ & $\begin{array}{l}\text { SBP } \\
\text { DBP }\end{array}$ & No & 5 \\
\hline
\end{tabular}


Table 1. Cont.

\begin{tabular}{|c|c|c|c|c|c|c|c|c|c|c|}
\hline $\begin{array}{l}\text { Author } \\
\text { Year }\end{array}$ & Design & Country & Population & Sex & Shift Work & $\begin{array}{l}\text { Sample Size } \\
\text { (SWs/DWs) }\end{array}$ & $\begin{array}{l}\text { Mean Age } \\
\text { (SWs/DWs) }\end{array}$ & Outcome & Outcome Adjustments & NOS \\
\hline $\begin{array}{c}\text { Kawada } \\
2014 \\
{[33]}\end{array}$ & CS & Japan & car manufacturing & M & $\begin{array}{c}\mathrm{R}+\mathrm{N} \\
\mathrm{R}-\mathrm{N}\end{array}$ & $\begin{array}{c}R+N 99 \\
R-N \text { 686/868 }\end{array}$ & $\begin{array}{c}\mathrm{R}+\mathrm{N} 44.5 \\
\mathrm{R}-\mathrm{N} 44.3 / 44.4\end{array}$ & $\begin{array}{l}\text { SBP } \\
\text { DBP }\end{array}$ & No & 5 \\
\hline $\begin{array}{c}\text { Kawakami } 1998 \\
{[34]}\end{array}$ & CS & Japan & $\begin{array}{l}\text { electrical } \\
\text { company }\end{array}$ & M & $\mathrm{R}+\mathrm{N}$ & $\begin{array}{l}\text { H } 161 / 123 \\
\text { A } 280 / 355 \\
\text { P } 186 / 178 \\
\text { L } 546 / 1053\end{array}$ & NotR & $\begin{array}{l}* \mathrm{SBP} \\
* \mathrm{DBP}\end{array}$ & $\begin{array}{c}\text { *age, obesity, exercise, } \\
\text { alcohol, } \\
\text { education }\end{array}$ & 8 \\
\hline $\begin{array}{c}\text { Knutsson } \\
1988 \\
{[35]}\end{array}$ & CS & Sweden & $\begin{array}{l}\text { paper and } \\
\text { cellulose plants }\end{array}$ & $\mathrm{M}$ & $\mathrm{R}+\mathrm{N}$ & $361 / 240$ & $43.2 / 44.8$ & $\begin{array}{l}\text { SBP } \\
\text { DBP }\end{array}$ & No & 4 \\
\hline $\begin{array}{c}\text { Kubo } \\
2013 \\
{[36]}\end{array}$ & $\begin{array}{c}\mathrm{CH} \\
(12.7 \mathrm{y})\end{array}$ & Japan & $\begin{array}{c}\text { industry } \\
\text { manufacturing }\end{array}$ & $\mathrm{M}$ & $\mathrm{R}+\mathrm{N}$ & $964 / 9209$ & $22.3 / 23.8$ & $\begin{array}{c}\text { SBP } \\
\text { DBP } \\
* \text { HTN }\end{array}$ & $\begin{array}{l}\text { * age, smoking, alcohol, } \\
\text { exercise, BP and BMI at } \\
\text { baseline and follow-up }\end{array}$ & 8 \\
\hline $\begin{array}{l}\text { Lang } \\
1988 \\
{[37]}\end{array}$ & CS & Senegal & $\begin{array}{l}\text { hotel, canning, } \\
\text { cotton printing, } \\
\text { tobacco, oil, } \\
\text { companies }\end{array}$ & $\mathrm{F} \& \mathrm{M}$ & NS & $396 / 900$ & $\begin{array}{c}\text { M } 39.3 \pm 9.7 \\
\text { F } 35.4 \pm 8.8\end{array}$ & $\begin{array}{l}* \text { SBP } \\
* \text { DBP }\end{array}$ & * age & 5 \\
\hline $\begin{array}{c}\text { Lercher } \\
1993 \\
{[38]}\end{array}$ & CS & Austria & rural community & $\mathrm{F} \& \mathrm{M}$ & $\mathrm{PN}$ & $22 / 147$ & [25-62] & $\begin{array}{l}\text { * SBP } \\
* \mathrm{DBP}\end{array}$ & $\begin{array}{c}\text { * age, sex, education, } \\
\text { smoking, BMI, other } \\
\text { occupational risk } \\
\text { factors }\end{array}$ & 8 \\
\hline $\begin{array}{c}\text { Lin } \\
2015 \\
{[39]}\end{array}$ & CS & Taiwan & $\begin{array}{l}\text { electronics } \\
\text { company }\end{array}$ & $\mathrm{F} \& \mathrm{M}$ & $\mathrm{RN}$ & $\begin{array}{c}\text { M 447/375 } \\
\text { F } 118 / 137\end{array}$ & $\begin{array}{c}\text { M } 31.5 / 33.8 \\
\text { F 32.5/31.7 }\end{array}$ & $\begin{array}{l}\text { SBP } \\
\text { DBP }\end{array}$ & No & 4 \\
\hline $\begin{array}{c}\text { Marqueze } \\
2013 \\
{[40]}\end{array}$ & CS & Brazil & truck drivers & $\mathrm{M}$ & $\mathrm{PN}$ & $31 / 26$ & $39.8 \pm 6.6$ & HTN & No & 5 \\
\hline $\begin{array}{l}\text { Nazri } \\
2008 \\
{[41]}\end{array}$ & CS & Malaysia & $\begin{array}{l}\text { semiconductors } \\
\text { factory }\end{array}$ & $\mathrm{M}$ & $\mathrm{R}+\mathrm{N}$ & $76 / 72$ & $31.60 / 32.32$ & ${ }^{*} \mathrm{HTN}$ & $\begin{array}{c}\text { * age, BMI, smoking, } \\
\text { exercise, } \\
\text { education, marital } \\
\text { status, job, working } \\
\text { hours and duration }\end{array}$ & 7 \\
\hline
\end{tabular}


Table 1. Cont.

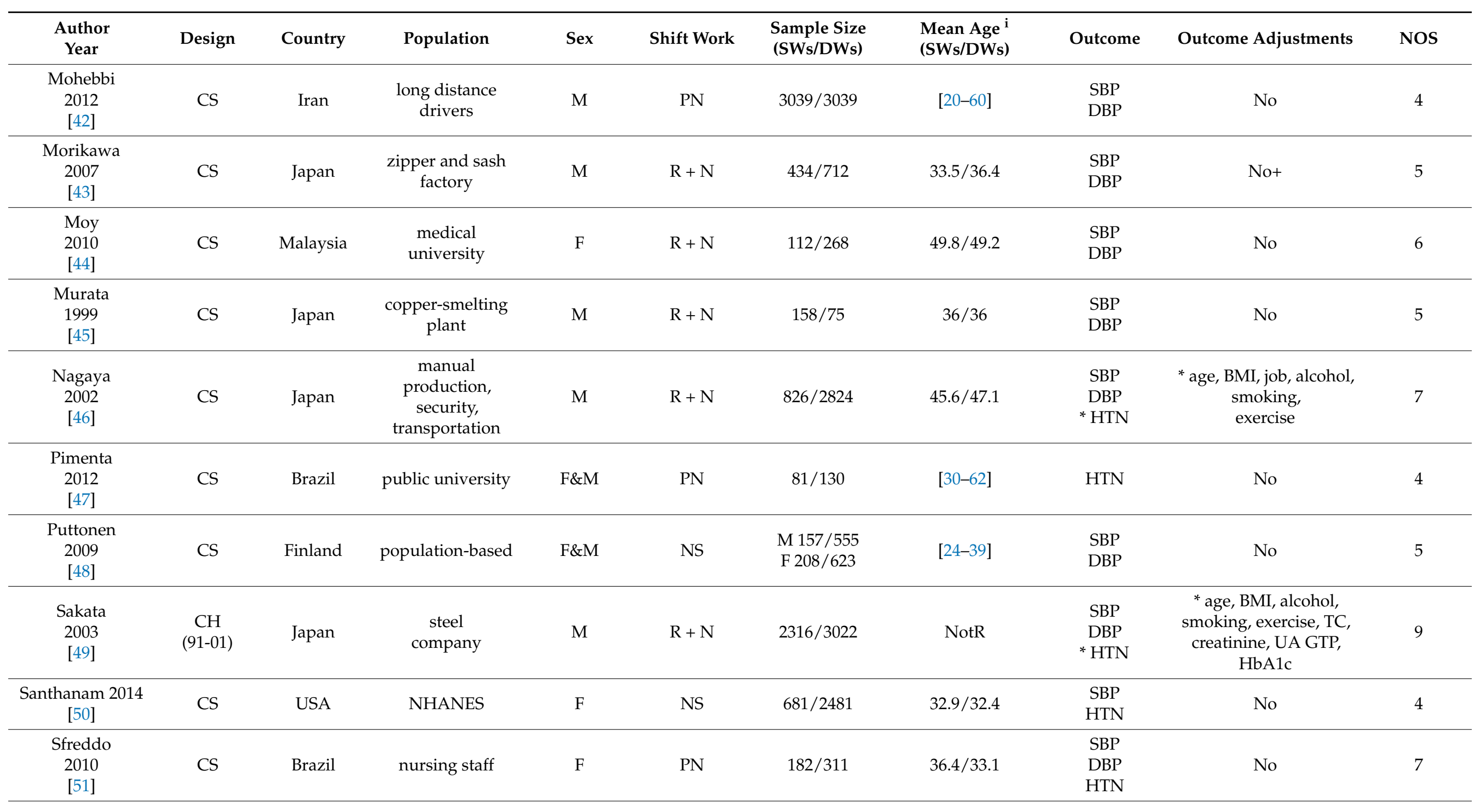


Table 1. Cont

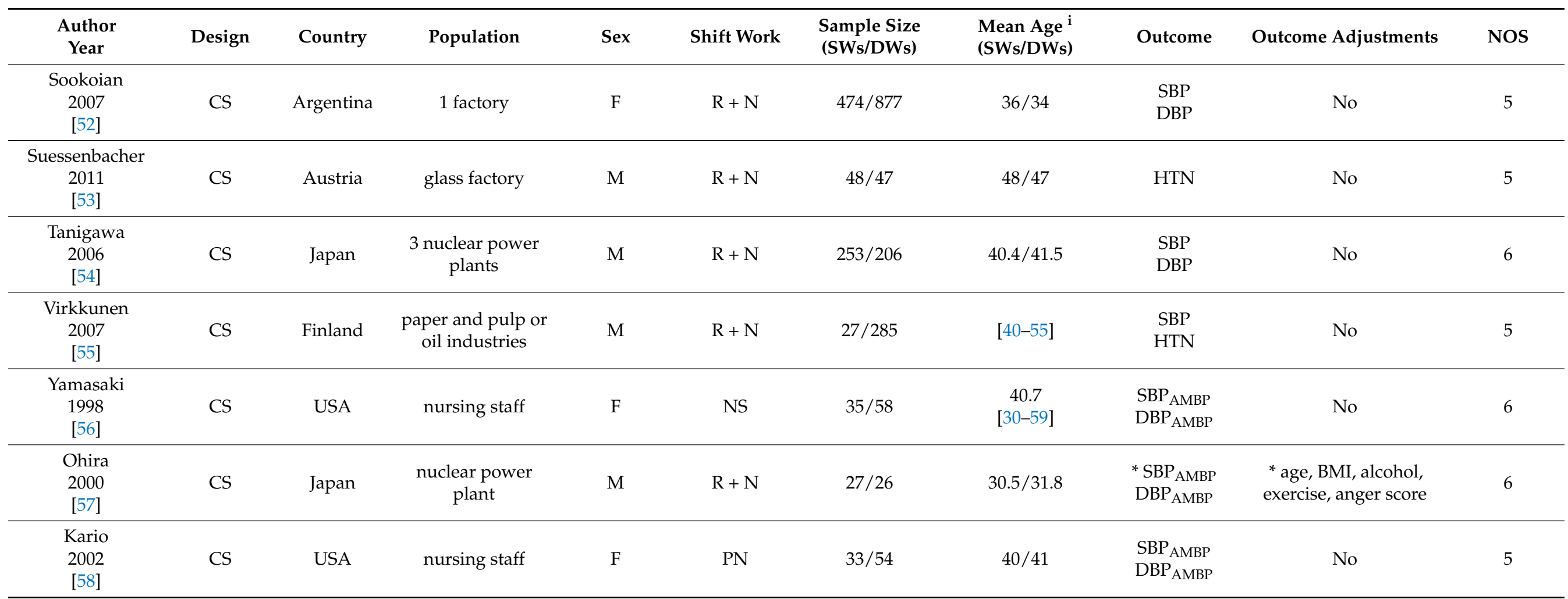

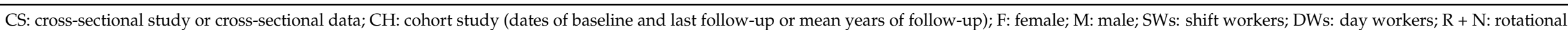

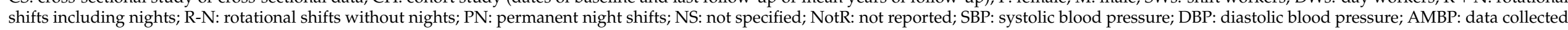

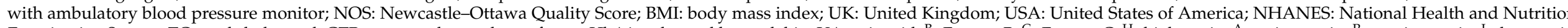

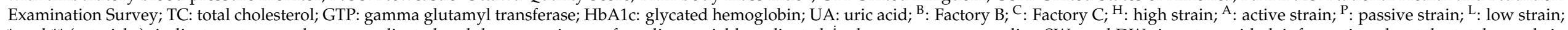

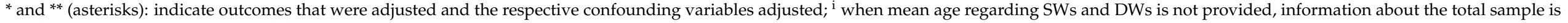
displayed both as mean \pm standard deviation or range (min-max). 
For exposure assessment, most studies used questionnaires or interviews $(n=35)$ and the remainder used company records $(n=10)$. The definition of SW was very heterogeneous. Given the original description of SW schedules, we categorized the shift workers according to the influence of work schedule in the night-time and, as a result, the potential impact on sleep and circadian system. Three categories emerged: permanent night shifts (PN; $n=14)$, rotational shifts including nights $(\mathrm{R}+\mathrm{N} ; n=28)$ and rotational shifts without nights $(\mathrm{R}-\mathrm{N} ; n=4)$. In some cases, the type of schedule was not well explicit $[14,22,28,37]$ or the population of SWs resulted from a combination of different schedules $[15,48,50,56]$. Such cases were labelled as a fourth category "Not Specified" (NS; $n=8)$. Of note, studies that included different types of SW (e.g., permanent night workers and rotational shifts including nights) compared to the same population of DWs were considered independent entries and included in independent meta-analyses. This provided segregate results according to the type of SW, with a more homogeneous exposure within groups.

Most studies provided more than one outcome of interest. A frequent combination was systolic BP and diastolic BP $(n=31)$, but also systolic BP, diastolic BP and HTN diagnosis $(n=8)$, with 4 studies accounting just for HTN and only 2 studies reporting systolic BP and HTN. Three studies provided data from ambulatory blood pressure monitoring [56-58]. Information regarding drug treatment with antihypertensive drugs was not reported or taken into consideration in most studies. A minority of studies had controlled the outcomes of interest for confounding factors $(n=13)$. Age was a ubiquitous adjusted variable. Other variables included lifestyle factors (e.g., smoking, alcohol and exercise) and occupational characteristics (e.g., job duration). Only one study [25] adjusted for sleep disturbances, whereas none controlled for sleep duration or deprivation, sleep quality or individual chronotype.

\subsection{Risk of Bias}

The Newcastle-Ottawa Scale (NOS) was used to evaluate the risk of bias of the included studies. The average NOS score was 5.6 points (median $=5$; interquartile range $=2.25$ ) with eleven studies scoring below 5 (low quality/high risk of bias) [9]. These eleven studies contributed to SBP and DBP results and only one for HTN. All included studies scored in the outcome and exposure ascertainment items since we excluded self-reported outcomes and exposure data derived from questionnaires or records. Therefore, the weakest dimension was comparability, with a minority of studies controlling the results of interest for confounding factors. All the included cohorts had an adequate follow-up period. The total NOS score for each included study is presented in Table 1. More details about the risk of bias of individual studies are shown in supplementary material (Table S3).

\subsection{Effect of Shift Work on Systolic Blood Pressure (SBP)}

Weighted mean differences and 95\% CI for systolic BP (SBP), according to the SW type, are shown in Figure 2. Permanent night work had the highest estimate, with a $2.52 \mathrm{mmHg}$ increase on SBP (95\% CI 0.75-4.29; $\mathrm{I}^{2}=91 \% ; 12$ studies; 29,923 participants). A positive effect was also found among rotational shifts without night work, with a $1.28 \mathrm{mmHg}$ increase (95\% CI 0.18-2.39; $\mathrm{I}^{2}=93 \% ; 4$ studies; 31,805 participants). Within the most common exposure, rotational shifts including night work (28 studies; 81,687 participants), the increase on SBP was $0.65 \mathrm{mmHg}\left(95 \%\right.$ CI 0.07-1.22; $\left.\mathrm{I}^{2}=69 \%\right)$. The "Not Specified" group had an estimate that did not reach statistical significance $(1.20 \mathrm{mmHg}$; $95 \%$ CI 0.15-2.55; $\mathrm{I}^{2}=79 \% ; 8$ studies; 10,548 participants). Subgroup differences were not statistically significant $(p=0.20)$ and there was no evidence of publication bias according to the Egger test $(p=0.418)$. Meta-regression analysis did not find a significant modifier effect on the mean difference of SBP for any of the covariates analyzed (sex, age, smoking and BMI) (see supplementary material for full results-Tables S4 and S5). 


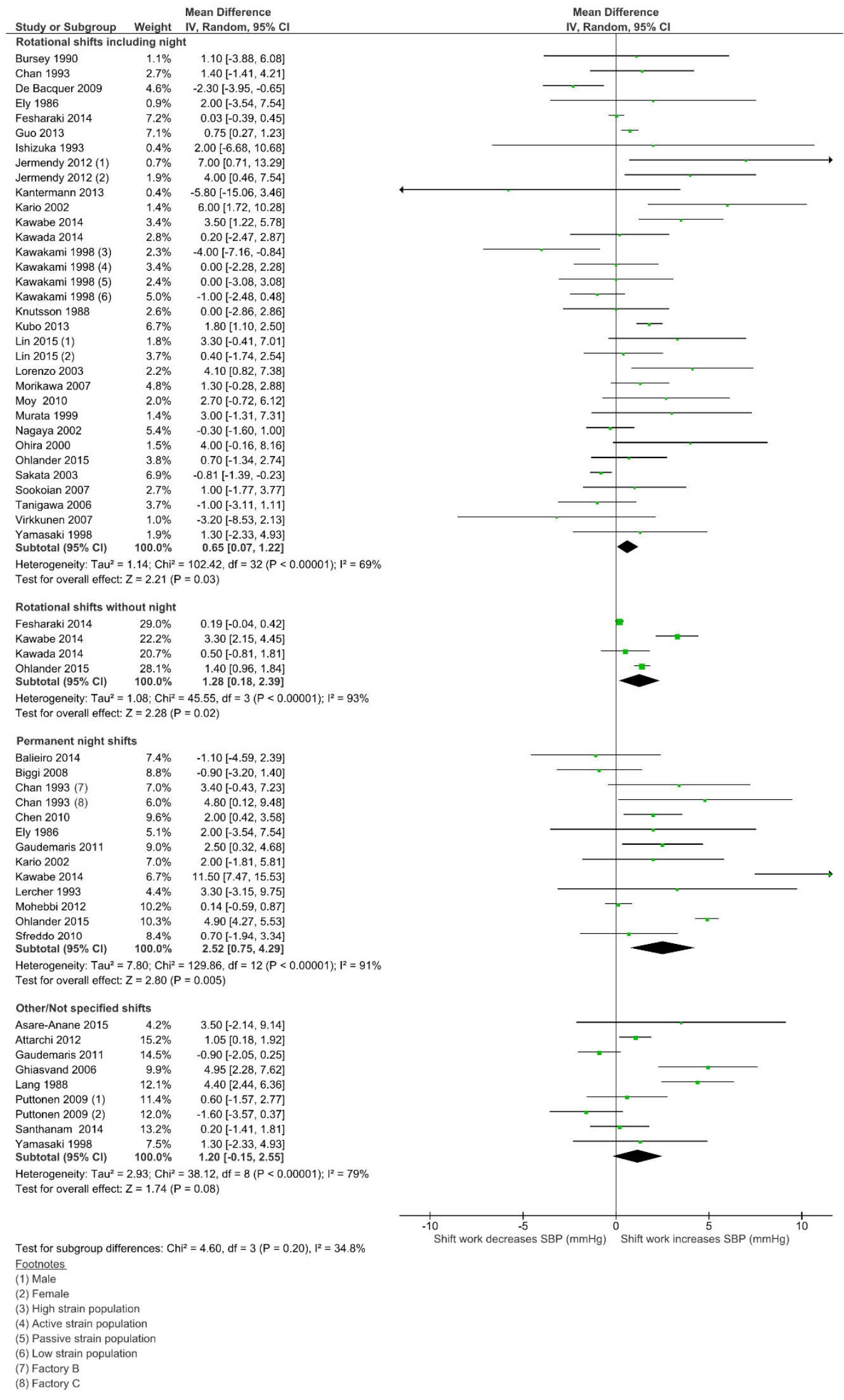

Figure 2. Forest plot showing the potential impact of the different shift work types in systolic blood pressure (SBP). 


\subsection{Effect of Shift Work on Diastolic Blood Pressure (DBP)}

Weighted mean differences and $95 \% \mathrm{CI}$ for diastolic BP (DBP), according to the SW type, are shown in Figure 3. As for SBP, the permanent night work had the highest estimate, with a $1.76 \mathrm{mmHg}$ increase on DBP $\left(95 \%\right.$ CI $0.41-3.12, \mathrm{I}^{2}=93 \%$; 12 studies; 29,923 participants). In fact, this was the only subgroup that reached statistical significance on DBP. As well as for SBP, the rotational shifts without nights was the second highest (0.60 mmHg; 95\% CI 0.24-1.43; $\mathrm{I}^{2}=92 \%$; 4 studies; 31,805 participants), followed by rotational shifts including night work $\left(0.12 \mathrm{mmHg} ; 95 \%\right.$ CI $0.31-0.54 ; \mathrm{I}^{2}=65 \% ; 25$ studies; 81,195 participants) and, finally, the "Not Specified" group $(0.22 \mathrm{mmHg}$; $95 \% \mathrm{CI} 0.68-1.12$; $\mathrm{I}^{2}=71 \% ; 7$ studies; 7385 participants). No subgroup differences were statistically significant $(p=0.13)$ and there was no evidence of publication bias (Egger test $p=0.447)$. Metaregression analysis did not find a significant modifier effect on the mean difference of DBP for any of the covariates analyzed (sex, age, smoking and BMI) (see supplementary material for full results-Tables S4 and S5).

\subsection{Effect of Shift Work on Hypertension (HTN)}

The pooled analysis showed that none of SW types were significantly associated with neither an increase nor a reduction in the risk for HTN diagnosis (Figure 4). The rotational shifts including night work, the most frequent SW type (8 studies; 33,716 participants), had the highest estimate with an increased risk of HTN by $26 \%$, however this was not statistically significant $\left(\mathrm{OR}=1.26 ; 95 \% \mathrm{CI} 0.94-1.69 ; \mathrm{I}^{2}=90 \%\right)$. Permanent night work revealed a neutral effect on HTN risk $\left(\mathrm{OR}=1.00 ; 95 \%\right.$ CI $0.80-1.27 ; \mathrm{I}^{2}=35 \% ; 6$ studies; 17,075 participants), as well as rotational shifts without nights ( $\mathrm{OR}=1.00 ; 95 \% \mathrm{CI} 0.88-1.15$; 1 study; 21,577participants) and the "Not Specified" group (OR = 0.83; 95\% CI 0.67-1.03; $\mathrm{I}^{2}=0 \% ; 2$ studies; 3586 participants). No subgroup differences were statistically significant $(p=0.16)$ and there was no evidence of publication bias (Egger test $p=0.957)$. 
Mean Difference Mean Difference

Study or Subgroup Weight IV, Random, $95 \% \mathrm{Cl}$ , Random, Rotational shifts including night

$\begin{array}{lrr}\text { Bursey 1990 } & 1.1 \% & -1.10[-4.92,2.72] \\ \text { Chan 1993 } & 2.2 \% & 0.00[-2.40,2.40] \\ \text { De Bacquer 2009 } & 5.0 \% & -2.80[-3.95,-1.65] \\ \text { Ely 1986 } & 0.5 \% & 2.00[-3.54,7.54] \\ \text { Fesharaki 2014 } & 6.8 \% & 0.19[-0.44,0.82] \\ \text { Guo 2013 } & 7.8 \% & 0.59[0.31,0.87] \\ \text { Ishizuka 1993 } & 0.6 \% & 0.30[-5.03,5.63] \\ \text { Jermendy 2012 (1) } & 1.1 \% & 0.00[-3.75,3.75] \\ \text { Jermendy 2012 (2) } & 2.6 \% & 1.00[-1.17,3.17] \\ \text { Kantermann 2013 } & 0.4 \% & -3.00[-9.28,3.28] \\ \text { Kawabe 2014 } & 3.6 \% & 1.20[-0.44,2.84] \\ \text { Kawada 2014 } & 2.9 \% & -0.10[-2.11,1.91] \\ \text { Kawakami 1998 (3) } & 3.1 \% & -1.00[-2.88,0.88] \\ \text { Kawakami 1998 (4) } & 2.0 \% & 2.00[-0.57,4.57] \\ \text { Kawakami 1998 (5) } & 4.7 \% & 0.00[-1.24,1.24] \\ \text { Kawakami 1998 (6) } & 1.6 \% & -2.00[-4.95,0.95] \\ \text { Knutsson 1988 } & 3.3 \% & -0.30[-2.09,1.49] \\ \text { Kubo 2013 } & 6.8 \% & -0.90[-1.54,-0.26] \\ \text { Lin 2015 (2) } & 2.1 \% & 3.30[0.83,5.77] \\ \text { Lin 2015(1) } & 4.3 \% & 0.00[-1.37,1.37] \\ \text { Lorenzo 2003 } & 2.6 \% & 0.80[-1.36,2.96] \\ \text { Morikawa 2007 } & 4.8 \% & -1.10[-2.33,0.13] \\ \text { Moy 2010 } & 4.2 \% & 1.10[-0.31,2.51] \\ \text { Murata 1999 } & 1.5 \% & 2.00[-1.11,5.11] \\ \text { Nagaya 2002 } & 6.0 \% & -0.20[-1.06,0.66] \\ \text { Ohira 2000 } & 1.2 \% & 1.00[-2.51,4.51] \\ \text { Ohlander 2015 } & 4.5 \% & 2.30[0.99,3.61] \\ \text { Sakata 2003 } & 7.4 \% & 0.22[-0.23,0.67] \\ \text { Sookoian 2007 } & 1.8 \% & 2.00[-0.77,4.77] \\ \text { Tanigawa 2006 } & 3.4 \% & -1.00[-2.76,0.76] \\ \text { Subtotal (95\% Cl) } & 100.0 \% & 0.12[-0.31,0.54]\end{array}$

$\begin{array}{lrr}\text { Tanigawa } 2006 & 3.4 \% & -1.00[-2.76,0.76 \\ \text { Subtotal }(95 \% \mathrm{Cl}) & 100.0 \% & 0.12[-0.31,0.54]\end{array}$

Heterogeneity: $\mathrm{Tau}^{2}=0.58 ; \mathrm{Chi}^{2}=83.06, \mathrm{df}=29(\mathrm{P}<0.00001) ; \mathrm{I}^{2}=65 \%$ Test for overall effect: $Z=0.54(P=0.59)$

Rotational shifts without night

Fesharaki $2014 \quad 28.1 \% \quad 0.10[-0.22,0.41]$

Kawabe $2014 \quad 22.9 \% \quad 0.40[-0.41,1.21]$

Kawada $2014 \quad 20.7 \% \quad 0.40[-0.59,1.39]$

$\begin{array}{lrr}\text { Ohlander } 2015 & 28.3 \% & 1.40[1.12,1.68] \\ \text { Subtotal }(95 \% \mathrm{Cl}) & 100.0 \% & \mathbf{0 . 6 0}[-0.24,1.43]\end{array}$

Heterogeneity: $\mathrm{Tau}^{2}=0.62 ; \mathrm{Chi}^{2}=38.47, \mathrm{df}=3(\mathrm{P}<0.00001) ; \mathrm{I}^{2}=92 \%$

Test for overall effect: $Z=1.40(P=0.16)$

Permanent night shifts

$\begin{array}{lrr}\text { Balieiro 2014 } & 7.6 \% & -1.00[-3.39,1.39] \\ \text { Biggi 2008 } & 9.1 \% & 0.30[-1.00,1.60] \\ \text { Chan 1993 (7) } & 7.1 \% & 3.60[0.89,6.31] \\ \text { Chan 1993 (8) } & 7.2 \% & 1.70[-0.99,4.39] \\ \text { Chen 2010 } & 9.4 \% & 2.00[0.99,3.01] \\ \text { Ely 1986 } & 3.7 \% & 0.00[-5.54,5.54] \\ \text { Gaudemaris 2011 } & 8.8 \% & 1.30[-0.24,2.84] \\ \text { Kario 2002 } & 7.1 \% & 0.00[-2.70,2.70] \\ \text { Kawabe 2014 } & 7.3 \% & 6.30[3.72,8.88] \\ \text { Lercher 1993 } & 4.4 \% & 3.90[-0.87,8.67] \\ \text { Mohebbi 2012 } & 9.8 \% & -0.29[-0.80,0.23] \\ \text { Ohlander 2015 } & 9.9 \% & 3.70[3.31,4.09] \\ \text { Sfreddo 2010 } & 8.3 \% & 1.90[-0.02,3.82] \\ \text { Subtotal (95\% Cl) } & 100.0 \% & 1.76[0.41,3.12] \\ \text { Tetol }\end{array}$

$\begin{array}{lrr}\text { Sfreddo } 2010 & 8.3 \% & 1.90[-0.02,3.82] \\ \text { Subtotal }(95 \% \mathrm{Cl}) & 100.0 \% & 1.76[0.41,3.12]\end{array}$

Heterogeneity: $\mathrm{Tau}^{2}=4.77 ; \mathrm{Chi}^{2}=177.04, \mathrm{df}=12(P<0.00001) ; \mathrm{I}^{2}=93 \%$

Test for overall effect: $Z=2.56(P=0.01)$

Other/Not specified shifts

$\begin{array}{lrr}\text { Asare-Anane 2015 } & 5.5 \% & 0.60[-2.71,3.91] \\ \text { Attarchi 2012 } & 17.6 \% & 1.08[0.21,1.95]\end{array}$

$\begin{array}{lrr}\text { Attarchi } 2012 & 17.6 \% & 1.08[0.21,1.95] \\ \text { Gaudemaris 2011 } & 19.2 \% & -0.60[-1.20,-0.00]\end{array}$

Ghiasvand $2006 \quad 11.8 \% \quad 2.56[0.82,4.30]$

Lang $1988 \quad 15.7 \% \quad 0.70[-0.44,1.84]$

Puttonen $2009(2) \quad 12.6 \% \quad-1.50[-3.11,0.11]$

Puttonen 2009 (1) $\quad 10.0 \% \quad-1.00[-3.06,1.06]$

$\begin{array}{lrr}\text { Yamasaki } 1998 & 7.6 \% & -0.10[-2.72,2.52\end{array}$

Heterogeneity: $\mathrm{Tau}^{2}=1.01 ; \mathrm{Chi}^{2}=23.79, \mathrm{df}=7(\mathrm{P}=0.001) ; \mathrm{I}^{2}=71 \%$

Test for overall effect: $Z=0.48(P=0.63)$

Test for subgroup differences: $\mathrm{Ch}^{2}=5.74, \mathrm{df}=3(\mathrm{P}=0.13), \mathrm{I}^{2}=47.7 \%$

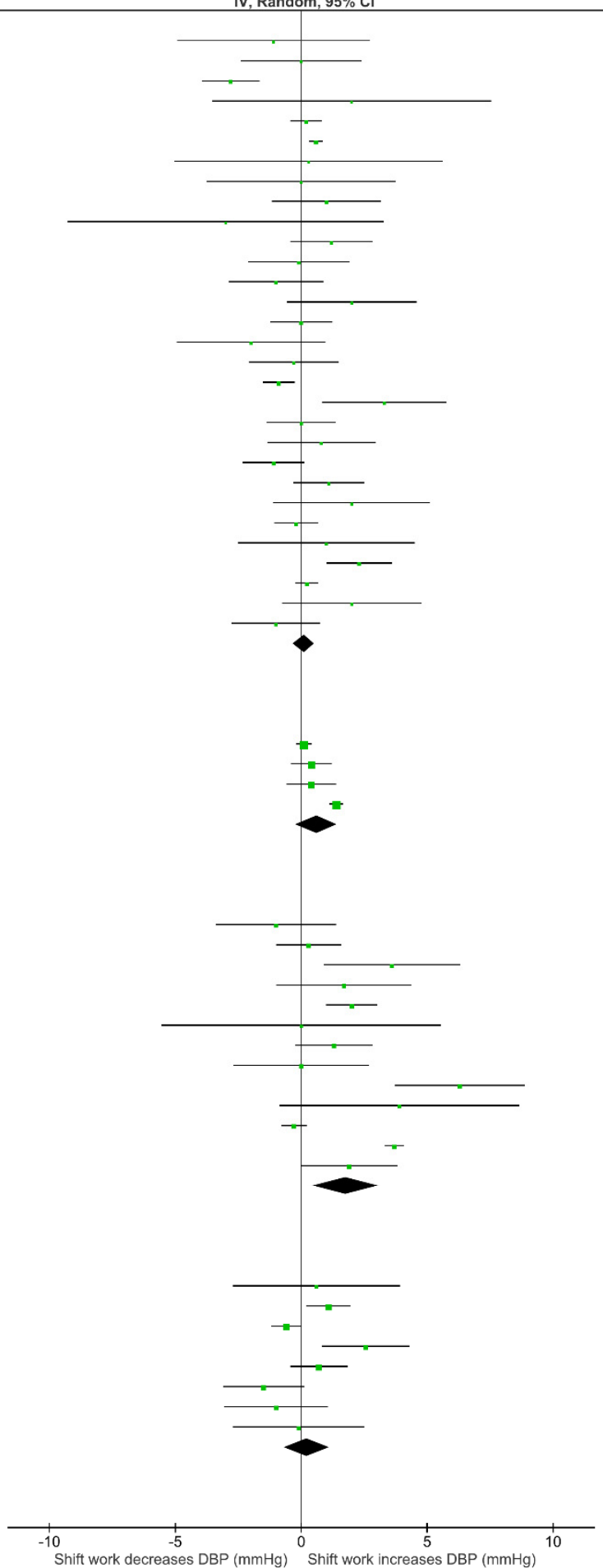

Footnotes

(1) Male

(2) Female

(3) High strain

(5) Passive strain

(6) Low strain

(7) Factory $B$

(8) Factory C

Figure 3. Forest plot showing the potential impact of the different shift work types in diastolic blood pressure (DBP). 
Odds Ratio

Study or Subgroup Weight IV, Random, $95 \% \mathrm{CI}$

Rotational shifts including night

Chan $1993 \quad 1.0 \%$

Kubo $2013 \quad 17.3 \%$

Ohlander $2015 \quad 10.6 \%$

Nagaya 2002 (1) $\quad 14.2 \%$

Nagaya $2002(2) \quad 10.1 \%$

Virkkunen $2007 \quad 7.6 \%$

Nagaya 2002 (3) $\quad 14.4 \%$

Sakata $2003 \quad 17.4 \%$

Suessenbacher $2011 \quad 5.3 \%$

Nazri 2008

Subtotal $(95 \% \mathrm{CI})$

$2.2 \%$

$100.0 \%$

$1.37[0.08,22.40]$

$1.85[1.68,2.03]$

$0.75[0.53,1.04]$

$1.20[0.66,2.16]$

$1.00[0.45,2.20]$

$1.62[1.17,2.24]$

$1.10[1.02,1.19]$

$1.70[0.60,4.84]$

$9.07[1.45,56.75]$

$1.26[0.94,1.68]$

作 ${ }^{2}=0.12 ; \mathrm{Chi}^{2}=89.23, \mathrm{df}=9(\mathrm{P}<0.00001) ;\left.\right|^{2}=90 \%$

Test for overall effect: $Z=1.57(P=0.12)$

Rotational shifts without night

Ohlander $2015 \quad 100.0 \%$

Subtotal $(95 \% \mathrm{Cl}) \quad 100.0 \%$

$1.00[0.88,1.15]$

$1.00[0.88,1.15]$

Heterogeneity: Not applicable

Test for overall effect: $Z=0.06(P=0.95)$

Permanent night shifts

$\begin{array}{lrr}\text { Biggi 2008 } & 28.2 \% & 0.90[0.67,1.20] \\ \text { Chan 1993 (4) } & 1.8 \% & 2.24[0.42,11.98] \\ \text { Chan } 1993(5) & 0.6 \% & 9.83[0.52,186.76] \\ \text { Ohlander 2015 } & 39.2 \% & 0.82[0.69,0.98] \\ \text { Marqueze 2013 } & 3.8 \% & 1.29[0.41,4.07] \\ \text { Pimenta 2011 } & 11.1 \% & 1.60[0.86,2.95] \\ \text { Sfreddo 2010 } & 15.3 \% & 1.12[0.69,1.84] \\ \text { Subtotal (95\% Cl) } & 100.0 \% & 1.00[0.80,1.27]\end{array}$

Heterogeneity: $\mathrm{Tau}^{2}=0.03 ; \mathrm{Chi}^{2}=9.22, \mathrm{df}=6(\mathrm{P}=0.16) ; \mathrm{I}^{2}=35 \%$

Test for overall effect: $Z=0.03(P=0.98)$

Other/Not specified shifts

$\begin{array}{lrc}\text { Ghiasvand 2006 } & 11.6 \% & 0.73[0.38,1.39] \\ \text { Santhanam 2014 } & 88.4 \% & 0.84[0.67,1.06] \\ \text { Subtotal }(95 \% \mathrm{CI}) & 100.0 \% & \mathbf{0 . 8 3}[0.67,1.03] \\ \text { Heterogeneity: } \mathrm{Tau}^{2}=0.00 ; \mathrm{Chi}^{2}=0.17, \mathrm{df}=1(\mathrm{P}=0.68) ; \mathrm{I}^{2}=0 \%\end{array}$

Test for overall effect: $Z=1.68(P=0.09)$

Test for subgroup differences: $\mathrm{Chi}^{2}=5.24, \mathrm{df}=3(\mathrm{P}=0.16), \mathrm{I}^{2}=42.7 \%$

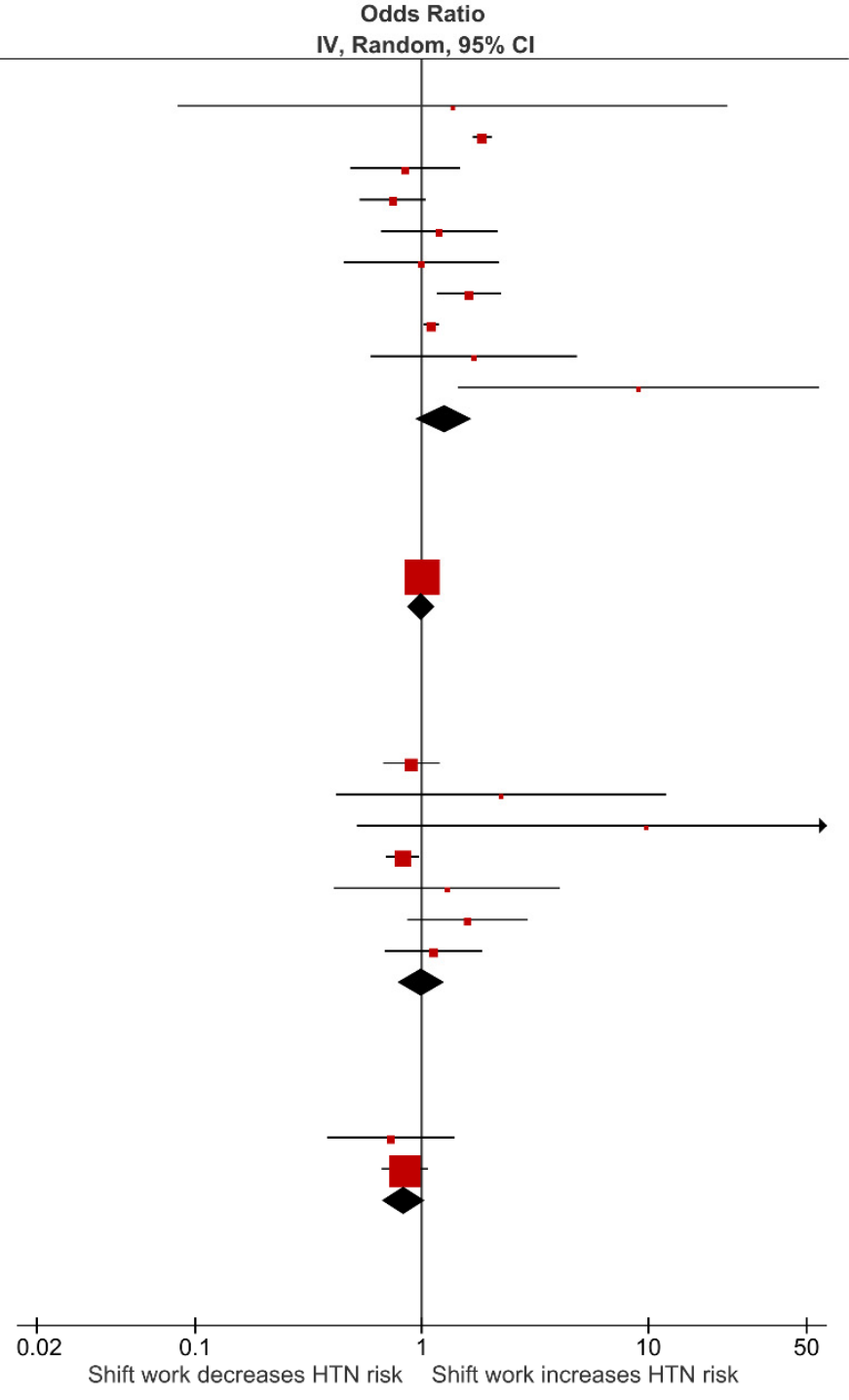

Footnotes

(1) $50-59$ years

(2) $30-39$ years

(3) 40-49 years

(4) Factory B

(5) Factory C

Odds Ratio IV, Random, $95 \%$ C
$0.85[0.48,1.48]$

Figure 4. Forest plot showing the potential impact of the different shift work types in hypertension (HTN).

\section{Discussion}

\subsection{Main Findings}

The main findings of this review, based on 45 independent studies which evaluated 46,345 shift workers against 70,907 day workers, were: (1) night workers had a statistically significant increase in both systolic and diastolic BP values; (2) rotational shift workers, both with and without night work, had a significant increase only in systolic BP; (3) the magnitude of the effect was small, ranging from 0.65 to $2.52 \mathrm{mmHg}$, and the larger upper bound of the pooled confidence intervals was $4.29 \mathrm{mmHg}$. This might seem as not clinically significant, however, it should be considered in susceptible populations continuously exposed over a considerable period of time, as a possible contributing factor for the development of HTN and/or for the need of more intensive drug treatment. Moreover, it was clearly demonstrated that the SW effect on BP values, although modest, is more consistent for SBP. This may be of special relevance considering that SBP has a major impact 
on CVD events [2]. Concerning HTN risk, we did not find a significant increase in any of the SW types assessed. This finding differs from the single previous meta-analysis in this topic [6], which found a greater risk among shift workers in cohort studies $(\mathrm{OR}=1.31 ; 95 \%$ CI 1.07-1.60) and an almost statistically significant increase among cross-sectional ones $(\mathrm{OR}=1.10 ; 95 \% \mathrm{CI} 1.00-1.20)$. Differences in these results can be explained by broader inclusion criteria in the previous review such as wider HTN definitions (e.g., metabolic syndrome thresholds of $130 / 85 \mathrm{mmHg}$ ), specific populations (e.g., sleep-disorder breathing patients and pregnant women) and different classifications of shift work types. Also, age is a major determinant for HTN [1] and the low average age of the included participants in our review (i.e., below 40 years) may have conditioned a low incidence of HTN, where differences between groups were not apparent. Since study subjects included in this systematic review were relatively young, the risk of hypertension in elderly shift workers may be increased. Further research will be needed concerning this aspect.

\subsection{Overall Limitations of Included Studies}

This is a systematic review with meta-analysis of study-level data, thus, our results are limited by the potential bias and intrinsic methodological limitations of the studies included. In fact, a major limitation of our review is related with the scarcity of adequate longitudinal data. This precludes not only the control for selection bias (the so-called healthy shift worker effect) but also the determination of a time sequence and a dose-response relationship which, in turn, hinders the assumption of causality. The "healthy shift worker effect" refers to the tendency for individuals with poorer health more likely quit shift work (survivor effect) or avoid it in the first place (hire effect) [59], resulting in an underestimation of the effects of shift work. On the other hand, the frequent higher payment for the same job, when performed outside the standard hours, can lead to a selection of lower socioeconomical status workers for SW. This is an important consideration given that lower socioeconomical conditions are associated to higher CVD risk [4] and few studies controlled for these variables. Furthermore, jobs which require SW frequently entail the performance of tasks with a higher physical strain. This alone may be associated to a higher risk for HTN, and few studies controlled for this specific issue. Indeed, one of the few which did, found a higher influence of physical strain than that of SW in the SBP and HTN [55]. Considering that the main mechanisms involved in the health consequences of shift work are unhealthy behaviors, sleep disturbance and circadian misalignment [59], only the first was assessed and controlled for in adjusted analyses. Sleep deprivation is commonly associated with SW and, in itself, represents a recognized cause for increased HTN risk [60] but almost no study evaluated and controlled for sleep duration and quality parameters. The same applies to the chronotype assessment, as a measurement of circadian entrainment, which can play a role in SW adaptation [61]. As diurnal creatures, human circadian system enables us to anticipate the light/dark cycle, ensuring optimal physiological functioning during the active day and restorative functioning during sleep. A healthy circadian rhythm of BP includes a considerable decrease during sleep, known as "dipping", that can be altered with shift work [62]. This confers biological plausibility for our results that revealed a higher risk of increased blood pressure among permanent night workers. It also highlights the importance of assessing BP through ambulatory blood pressure monitoring given the high CVD prognostic value of sleep-time BP [1].

\subsection{Strengths and Limitations}

To the best of our knowledge, this is the first systematic review with meta-analysis that assessed the impact of different types of SW on BP values, both systolic and diastolic. This is of special interest since CVD events have a continuous and proportional relationship with BP values [2]. Furthermore, this approach allowed the inclusion of studies which main outcome was not hypertension, but nevertheless provided BP measurements. As for HTN risk assessment, we assumed a strict and conservative approach by only considering the current HTN thresholds and excluding self-reported outcomes. Another innovative 
aspect was the division in specific types of SW, according to night-time work. This aimed to counteract the notoriously heterogenous nature of the SW definition and operationalization, allowing for more homogenous exposed groups concerning the circadian system and more precise results. Moreover, this strategy allowed for the same study providing data for more than one meta-analysis.

On the other hand, when we segregated the results into SW types, some groups resulted in too few studies. High levels of heterogeneity among pooled results were found. This may be due to the wide heterogeneity in the work settings and tasks performed in the included studies. In fact, although we have tried to mitigate the SW variability, even our SW types may encompass different working times, schemes, speed and direction of rotation. Additionally, the duration and intensity of the SW exposure (e.g., average number of shifts) may be implicated, since most studies did not provide any information about these features. Another possible limitation is a geographic bias, with almost half of the studies developed in Asia.

\section{Conclusions}

There is sufficient evidence for a potential link between permanent night shift work and an increase in blood pressure values. Regarding rotational shift work, both including nights or not, the evidence is only for an increment in systolic BP. As for hypertension, no increased risk was found. Although the effect on BP values was rather small, this can be of special interest in borderline situations or in susceptible populations with concurrent cardiovascular risk factors. Occupational health services may play an important role in limiting shift work health consequences by promoting healthy behaviors, while closely monitoring the more vulnerable workers. Considerations about circadian human physiology could support the design of least detrimental work schedules and select more adequate workers for certain shifts, according to their own individual chronotype. To accurately define the impact of shift work on blood pressure, interventional and longitudinal studies with appropriate follow-up are needed, which should include comprehensive shift work descriptions, continuous BP monitoring and, also, adjustment for relevant lifestyle, occupational and sleep parameters.

Supplementary Materials: The following are available online at https: / www.mdpi.com/article / 10.3390/ijerph18136738/s1. Figure S1: Funnel plots and $p$-value (for Egger test) for each outcome; Table S1: Search strategy; Table S2: Key studies excluded at full-text stage, with reasons; Table S3: Newcastle-Ottawa Quality Assessment Score (NOS). Table S4: Results from univariate meta-regression analysis. Table S5. Results from multivariate meta-regression analysis.

Author Contributions: S.G.M. created the concept of the study, searched the articles and took the lead in writing the manuscript. S.G.M. and C.F. wrote the study protocol, performed article screening, data extraction, analysis and risk of bias assessment. D.C. contributed to the study design, solved disagreements, performed the statistical analyses and coordinated database search and extraction. S.G.M., C.F., T.P., C.S.M. and D.C. were involved in the results interpretation, discussion and critically revised the manuscript. All authors have read and agreed to the published version of the manuscript.

Funding: This work was supported by the Ph.D. research Grant PDE/BDE/127787/2016 from Fundação para a Ciência e Tecnologia (FCT) /Fundo Social Europeu.

Institutional Review Board Statement: Ethical review and approval were waived for this research since data extracted and analyzed was from already published studies.

Informed Consent Statement: Participant's consent was waived for this research since data extracted and analyzed was from already published studies.

Acknowledgments: The authors would like to thank Joana Alarcão for the support in the electronic search strategy and also Sofia Amador and André Silva for papers retrieval.

Conflicts of Interest: The authors declare no conflict of interest. 


\section{References}

1. Williams, B.; Mancia, G.; Spiering, W.; Agabiti Rosei, E.; Azizi, M.; Burnier, M.; Clement, D.L.; Coca, A.; de Simone, G.; Dominiczak, A.; et al. 2018 ESC/ESH Guidelines for the Management of Arterial Hypertension. Eur. Heart J. 2018, 39, 3021-3104. [CrossRef]

2. $\quad$ Flint, A.C.; Conell, C.; Ren, X.; Banki, N.M.; Chan, S.L.; Rao, V.A.; Melles, R.B.; Bhatt, D.L. Effect of Systolic and Diastolic Blood Pressure on Cardiovascular Outcomes. N. Engl. J. Med. 2019, 381, 243-251. [CrossRef]

3. Ettehad, D.; Emdin, C.A.; Kiran, A.; Anderson, S.G.; Callender, T.; Emberson, J.; Chalmers, J.; Rodgers, A.; Rahimi, K. Blood Pressure Lowering for Prevention of Cardiovascular Disease and Death: A Systematic Review and Meta-Analysis. Lancet 2016, 387, 957-967. [CrossRef]

4. Piepoli, M.F.; Hoes, A.W.; Agewall, S.; Albus, C.; Brotons, C.; Catapano, A.L.; Cooney, M.-T.; Corrà, U.; Cosyns, B.; Deaton, C.; et al. 2016 European Guidelines on Cardiovascular Disease Prevention in Clinical Practice. Eur. Heart J. 2016, 37, 2315-2381. [CrossRef] [PubMed]

5. Messenger, J. Working Time and the Future of Work; International Labour Organization: Geneva, Switzerland, 2018.

6. Manohar, S.; Thongprayoon, C.; Cheungpasitporn, W.; Mao, M.A.; Herrmann, S.M. Associations of Rotational Shift Work and Night Shift Status with Hypertension: A Systematic Review and Meta-Analysis. J. Hypertens. 2017, 35, 1-9. [CrossRef] [PubMed]

7. Moher, D.; Liberati, A.; Tetzlaff, J.; Altman, D.G. Preferred Reporting Items for Systematic Reviews and Meta-Analyses: The PRISMA Statement. PLoS Med. 2009, 6, e1000097. [CrossRef]

8. Herzog, R.; Álvarez-Pasquin, M.J.; Díaz, C.; del Barrio, J.L.; Estrada, J.M.; Gil, Á. Are Healthcare Workers' Intentions to Vaccinate Related to Their Knowledge, Beliefs and Attitudes? A Systematic Review. BMC Public Health 2013, 13, 154. [CrossRef]

9. Luchini, C.; Stubbs, B.; Solmi, M.; Veronese, N. Assessing the Quality of Studies in Meta-Analyses: Advantages and Limitations of the Newcastle Ottawa Scale. World J. Meta Anal. 2017, 5, 80. [CrossRef]

10. Torquati, L.; Mielke, G.I.; Brown, W.J.; Kolbe-Alexander, T. Shift Work and the Risk of Cardiovascular Disease. A Systematic Review and Meta-Analysis Including Dose-Response Relationship. Scand. J. Work Environ. Health 2018, 44, 229-238. [CrossRef]

11. Higgins, J.P.T.; Green, S. (Eds.) Cochrane Handbook for Systematic Reviews of Interventions Version 5.1.0 [updated March 2011]. The Cochrane Collaboration, 2011. Available online: www.handbook.cochrane.org (accessed on 5 June 2021).

12. Lau, J.; Ioannidis, J.P.A.; Schmid, C.H. Quantitative Synthesis in Systematic Reviews. Ann. Intern. Med. 1997, 127, 820-826. [CrossRef]

13. Bray, E.P.; Holder, R.; Mant, J.; McManus, R.J. Does Self-Monitoring Reduce Blood Pressure? Meta-Analysis with Meta-Regression of Randomized Controlled Trials. Ann. Med. 2010, 42, 371-386. [CrossRef] [PubMed]

14. Asare-Anane, H.; Abdul-Latif, A.; Ofori, E.K.; Abdul-Rahman, M.; Amanquah, S.D. Shift Work and the Risk of Cardiovascular Disease among Workers in Cocoa Processing Company, Tema. BMC Res. Notes 2015, 8, 4-9. [CrossRef]

15. Attarchi, M.; Dehghan, F.; Safakhah, F.; Nojomi, M.; Mohammadi, S. Effect of Exposure to Occupational Noise and Shift Working on Blood Pressure in Rubber Manufacturing Company Workers. Ind. Health 2012, 50, 205-213. [CrossRef] [PubMed]

16. Balieiro, L.C.; Rossato, L.T.; Waterhouse, J.; Paim, S.L.; Mota, M.C.; Crispim, C.A. Nutritional Status and Eating Habits of Bus Drivers during the Day and Night. Chronobiol. Int. 2014, 31, 1123-1129. [CrossRef] [PubMed]

17. Biggi, N.; Consonni, D.; Galluzzo, V.; Sogliani, M.; Costa, G. Metabolic Syndrome in Permanent Night Workers. Chronobiol. Int. 2008, 25, 443-454. [CrossRef]

18. Bursey, R.G. A Cardiovascular Study of Shift Workers with Respect to Coronary Artery Disease Risk Factor Prevalence. J. Soc. Occup. Med. 1990, 40, 65-67. [CrossRef] [PubMed]

19. Chan, O.Y.; Gan, S.L.; Yeo, M.H. Study on the Health of Female Electronics Workers on 12 Hour Shifts. Occup. Med. 1993, 43, 143-148. [CrossRef] [PubMed]

20. Chen, J.D.; Lin, Y.C.; Hsiao, S.T. Obesity and High Blood Pressure of 12-Hour Night Shift Female Clean-Room Workers. Chronobiol. Int. 2010, 27, 334-344. [CrossRef]

21. De Bacquer, D.; van Risseghem, M.; Clays, E.; Kittel, F.; de Backer, G.; Braeckman, L. Rotating Shift Work and the Metabolic Syndrome: A Prospective Study. Int. J. Epidemiol. 2009, 38, 848-854. [CrossRef]

22. De Gaudemaris, R.; Levant, A.; Ehlinger, V.; Hérin, F.; Lepage, B.; Soulat, J.M.; Sobaszek, A.; Kelly-Irving, M.; Lang, T. Blood Pressure and Working Conditions in Hospital Nurses and Nursing Assistants. The ORSOSA Study. Arch. Cardiovasc. Dis. 2011, 104, 97-103. [CrossRef] [PubMed]

23. Di Lorenzo, L.; de Pergola, G.; Zocchetti, C.; L'Abbate, N.; Basso, A.; Pannacciulli, N.; Cignarelli, M.; Giorgino, R.; Soleo, L. Effect of Shift Work on Body Mass Index: Results of a Study Performed in 319 Glucose-Tolerant Men Working in a Southern Italian Industry. Int. J. Obes. 2003, 27, 1353-1358. [CrossRef] [PubMed]

24. Ely, D.L.; Mostardi, R.A. The Effect of Recent Life Events Stress, Life Assets, and Temperament Pattern on Cardiovascular Risk Factors for Akron City Police Officers. J. Hum. Stress 1986, 12, 77-91. [CrossRef]

25. Ohlander, J.; Keskin, M.C.; Stork, J.; Radon, K. Shift Work and Hypertension: Prevalence and Analysis of Disease Pathways in a German Car Manufacturing Company. Am. J. Ind. Med. 2015, 58, 549-560. [CrossRef]

26. Gholami-Fesharaki, M.; Kazemnejad, A.; Zayeri, F.; Rowzati, M.; Sanati, J.; Akbari, H. Multicenter Historical Cohort Study of the Relationship between Shift Work and Blood Pressure. ARYA Atheroscler. 2014, 10, 287-291.

27. Guo, Y.; Liu, Y.; Huang, X.; Rong, Y.; He, M.; Wang, Y.; Yuan, J.; Wu, T.; Chen, W. The Effects of Shift Work on Sleeping Quality, Hypertension and Diabetes in Retired Workers. PLoS ONE 2013, 8, e71107. [CrossRef] 
28. Ghiasvand, M.; Heshmat, R.; Golpira, R.; Haghpanah, V.; Soleimani, A.; Shoushtarizadeh, P.; Tavangar, S.M.; Larijani, B. Shift Working and Risk of Lipid Disorders: A Cross-Sectional Study. Lipids Health Dis. 2006, 5, 9. [CrossRef] [PubMed]

29. Ishizuka, T.; Furuya, Y. Influence of Meals and Night Shifts on Health. J. Med. Syst. 1993, 17, 201-206. [CrossRef]

30. Jermendy, G.; Nadas, J.; Hegyi, I.; Vasas, I.; Hidvegi, T. Assessment of Cardiometabolic Risk among Shift Workers in Hungary. Health Qual. Life Outcomes 2012, 10, 18. [CrossRef] [PubMed]

31. Kantermann, T.; Duboutay, F.; Haubruge, D.; Kerkhofs, M.; Schmidt-Trucksäss, A.; Skene, D.J. Atherosclerotic Risk and Social Jetlag in Rotating Shift-Workers: First Evidence from a Pilot Study. Work 2013, 46, 273-282. [CrossRef]

32. Kawabe, Y.; Nakamura, Y.; Kikuchi, S.; Murakami, Y.; Tanaka, T.; Takebayashi, T.; Okayama, A.; Miura, K.; Okamura, T.; Ueshima, H. Relationship between Shift Work and Clustering of the Metabolic Syndrome Diagnostic Components. J. Atheroscler. Thromb. 2014, 21, 703-711. [CrossRef]

33. Kawada, T.; Otsuka, T. Effect of Shift Work on the Development of Metabolic Syndrome After 3 Years in Japanese Male Workers. Arch. Environ. Occup. Health 2014, 69, 55-61. [CrossRef]

34. Kawakami, N.; Haratani, T.; Araki, S. Job Strain and Arterial Blood Pressure, Serum Cholesterol, and Smoking as Risk Factors for Coronary Heart Disease in Japan. Int. Arch. Occup. Environ. Health 1998, 71, 429-432. [CrossRef] [PubMed]

35. Knutsson, A.; Akerstedt, T.; Jonsson, B.G. Prevalence of Risk-Factors for Coronary-Artery Disease among Day and Shift Workers. Scand. J. Work Environ. Health 1988, 14, 317-321. [CrossRef]

36. Kubo, T.; Fujino, Y.; Nakamura, T.; Kunimoto, M.; Tabata, H.; Tsuchiya, T.; Kadowaki, K.; Odoi, H.; Oyama, I.; Matsuda, S. An Industry-Based Cohort Study of the Association Between Weight Gain and Hypertension Risk Among Rotating Shift Workers. J. Occup. Environ. Med. 2013, 55, 1041-1045. [CrossRef]

37. Lang, T.; Pariente, P.; Salem, G.; Tap, D. Social, Professional Conditions and Arterial Hypertension: An Epidemiological Study in Dakar, Senegal. J. Hypertens. 1988, 6, 271-276. [CrossRef]

38. Lercher, P.; Hortnagl, J.; Kofler, W.W. Work Noise Annoyance and Blood Pressure: Combined Effects with Stressful Working Conditions. Int. Arch. Occup. Environ. Health 1993, 65, 23-28. [CrossRef] [PubMed]

39. Lin, Y.C.; Hsieh, I.C.; Chen, P.C. Utilizing the Metabolic Syndrome Component Count in Workers' Health Surveillance: An Example of Day-Time vs. Day-Night Rotating Shift Workers. Int. J. Occup. Med. Environ. Health 2015, 28, 675-688. [CrossRef]

40. Marqueze, E.C.; Ulhoa, M.A.; Moreno, C.R.C. Effects of Irregular-Shift Work and Physical Activit on Cardiovascular Risk Factors in Truck Drivers. Rev. Saude Publica 2013, 47, 497-505. [CrossRef]

41. Nazri, S.M.; Tengku, M.A.; Winn, T. The Association of Shift Work and Hypertension among Male Factory Workers in Kota Bharu, Kelantan, Malaysia. Southeast Asian J. Trop. Med. Public Health 2008, 39, 176-183. [PubMed]

42. Mohebbi, I.; Shateri, K.; Seyedmohammadzad, M. The Relationship between Working Schedule Patterns and the Markers of the Metabolic Syndrome: Comparison of Shift Workers with Day Workers. Int. J. Occup. Med. Environ. Health 2012, 25, 383-391. [CrossRef]

43. Morikawa, Y.; Nakagawa, H.; Miura, K.; Soyama, Y.; Ishizaki, M.; Kido, T.; Naruse, Y.; Suwazono, Y.; Nogawa, K. Effect of Shift Work on Body Mass Index and Metabolic Parameters. Scand. J. Work Environ. Health 2007, 33, 45-50. [CrossRef] [PubMed]

44. Moy, F.M.; Hoe, V.C.W.; Tan, C.P.L.; Rosmawati, M. Cardiovascular Risks among Shift and Non-Shift Workers in a Public Medical Centre in Kuala Lumpur. J. Health Transl. Med. 2010, 13, 45-49.

45. Murata, K.; Yano, E.; Shinozaki, T. Impact of Shift Work on Cardiovascular Functions in a 10-Year Follow-up Study. Scand. J. Work Environ. Health 1999, 25, 272-277. [CrossRef]

46. Nagaya, T.; Yoshida, H.; Takahashi, H.; Kawai, M. Markers of Insulin Resistance in Day and Shift Workers Aged 30-59 Years. Int. Arch. Occup. Environ. Health 2002, 75, 562-568. [CrossRef] [PubMed]

47. Pimenta, A.M.; Kac, G.; e Souza, R.R.C.; de Barros Almeida Ferreira, L.M.; de Fátima Silqueira, S.M. Night-Shift Work and Cardiovascular Risk among Employees of a Public University. Rev. Assoc. Med. Bras. 2012, 58, 168-177. [CrossRef]

48. Puttonen, S.; Kivimäki, M.; Elovainio, M.; Pulkki-Råback, L.; Hintsanen, M.; Vahtera, J.; Telama, R.; Juonala, M.; Viikari, J.S.A.; Raitakari, O.T.; et al. Shift Work in Young Adults and Carotid Artery Intima-Media Thickness: The Cardiovascular Risk in Young Finns Study. Atherosclerosis 2009, 205, 608-613. [CrossRef]

49. Sakata, K.; Suwazono, Y.; Harada, H.; Okubo, Y.; Kobayashi, E.; Nogawa, K. The Relationship Between Shift Work and the Onset of Hypertension in Male Japanese Workers. J. Occup. Environ. Med. 2003, 45, 1002-1006. [CrossRef]

50. Santhanam, P.; Driscoll, H.K.; Gress, T.W.; Khthir, R. Metabolic Disease and Shift Work: Is There an Association? An Analysis of NHANES Data for 2007-2008. Occup. Environ. Med. 2014, 71, 661-662. [CrossRef]

51. Sfreddo, C.; Fuchs, S.C.; Merlo, A.R.; Fuchs, F.D. Shift Work Is Not Associated with High Blood Pressure or Prevalence of Hypertension. PLoS ONE 2010, 5, e15250. [CrossRef]

52. Sookoian, S.; Gemma, C.; Fernández Gianotti, T.; Burgueño, A.; Alvarez, A.; González, C.D.; Pirola, C.J. Effects of Rotating Shift Work on Biomarkers of Metabolic Syndrome and Inflammation. J. Intern. Med. 2007, 261, 285-292. [CrossRef]

53. Suessenbacher, A.; Potocnik, M.; Dörler, J.; Fluckinger, G.; Wanitschek, M.; Pachinger, O.; Frick, M.; Alber, H.F. Comparison of Peripheral Endothelial Function in Shift Versus Nonshift Workers. Am. J. Cardiol. 2011, 107, 945-948. [CrossRef]

54. Tanigawa, T.; Muraki, I.; Umesawa, M.; Tachibana, N.; Noda, H.; Takahashi, M.; Mutou, K.; Kage, Y.; Smith, L.; Iso, H. SleepDisordered Breathing and Blood Pressure Levels Among Shift and Day Workers. Am. J. Hypertens. 2006, 19, 346-351. [CrossRef] [PubMed] 
55. Virkkunen, H.; Härmä, M.; Kauppinen, T.; Tenkanen, L. Shift Work, Occupational Noise and Physical Workload with Ensuing Development of Blood Pressure and Their Joint Effect on the Risk of Coronary Heart Disease. Scand. J. Work Environ. Health 2007, 33, 425-434. [CrossRef]

56. Yamasaki, F.; Schwartz, J.E.; Gerber, L.M.; Warren, K.; Pickering, T.G. Impact of Shift Work and Race/Ethnicity on the Diurnal Rhythm of Blood Pressure and Catecholamines. Hypertension 1998, 32, 417-423. [CrossRef]

57. Ohira, T.; Tanigawa, T.; Iso, H.; Odagiri, Y.; Takamiya, T.; Shimomitsu, T.; Hayano, J.; Shimamoto, T. Effects of Shift Work on 24-Hour Ambulatory Blood Pressure and Its Variability among Japanese Workers. Scand. J. Work Environ. Health 2000, 26, 421-426. [CrossRef] [PubMed]

58. Kario, K.; Schwartz, J.E.; Gerin, W.; Robayo, N.; Maceo, E.; Pickering, T.G. Psychological and Physical Stress-Induced Cardiovascular Reactivity and Diurnal Blood Pressure Variation in Women with Different Work Shifts. Hypertens Res. 2002, 25, 543-551. [CrossRef] [PubMed]

59. Moreno, C.R.C.; Marqueze, E.C.; Sargent, C.; Wright, K.P., Jr.; Ferguson, S.A.; Tucker, P. Working Time Society Consensus Statements: Evidence-Based Effects of Shift Work on Physical and Mental Health. Ind. Health 2019, 57, 139-157. [CrossRef] [PubMed]

60. Makarem, N.; Shechter, A.; Carnethon, M.R.; Mullington, J.M.; Hall, M.H.; Abdalla, M. Sleep Duration and Blood Pressure: Recent Advances and Future Directions. Curr. Hypertens. Rep. 2019, 21. [CrossRef] [PubMed]

61. Juda, M.; Vetter, C.; Roenneberg, T. Chronotype Modulates Sleep Duration, Sleep Quality, and Social Jet Lag in Shift-Workers. J. Biol. Rhythm. 2013, 28, 141-151. [CrossRef]

62. Chellappa, S.L.; Vujovic, N.; Williams, J.S.; Scheer, F.A.J.L. Impact of Circadian Disruption on Cardiovascular Function and Disease. Trends Endocrinol. Metab. 2019, 30, 767-779. [CrossRef] 